\title{
XIX. On the possibility of accounting for the continuance of recurring changes in the universe, consistently with the tendency to temperature- equilibrium
}

\section{S. Tolver Preston}

To cite this article: S. Tolver Preston (1879) XIX. On the possibility of accounting for the continuance of recurring changes in the universe, consistently with the tendency to temperature-equilibrium , Philosophical Magazine Series 5, 8:47, 152-163, DOI: 10.1080/14786447908639666

To link to this article: http://dx.doi.org/10.1080/14786447908639666

$$
\text { 曲 Published online: } 13 \text { May } 2009 .
$$

Submit your article to this journal ¿

\section{山ll Article views: 2}

Q View related articles ¿ 
the smallest portion of any increase, must fall upon the part $\mathrm{A} \mathrm{B}$; and this we in fact learn from the above consideration. That I have therein taken no account of the action of neighbouring current-threads makes no difference; for the result of this action is, for A B exactly as for B C, that the molecular magnets are brought into a position a little nearer the perpendicular to the plane of the plate.

In Beetz's experiment the iron-wire spiral to be magnetized was inside the magnetizing copper spiral, so that the turns of the one were parallel to those of the other. Consequently the molecules were more or less approximately so placed that (supposing the windings horizontal and the current flowing in the copper in the direction of motion of the hands of a watch) all the north poles pointed downward. The principal currerit, on the other hand, called forth a circular magnetizing; therefore in the portions of wire belonging to the front half of the iron spiral, although the principal current flowed in the direction in which the hands of a watch move, the north poles in the anterior semicylinders into wh $\mathrm{ch}$ each portion of the wire can be resolved were directed more or less upward, in the posterior (inner) downward. Conversely, in the portions of wire of the hinder half-spiral, the north poles in the posterior (outer) semicylinders were directed upward, and downward in the anterior ones. If, then, the magnetizing force of the principal current is not very little in comparison with the other, half of the current-threads present a stronger resistance than before the transverse magnetizing, the other half a weaker one; thus the total resistance remains nearly unaltered. The nogative result of Beetz's experiment is therefore not surprising.

[To be continued.]

XIX. On the Possibility of accounting for the Continuance of Recurring Changes in the Universe, consistently with the Tendency to Temperature-Equilibrium. By S. Tolver Preston*.

TTHE idea of the ultimate final cessation of all activity and 1 life in the universe has been contemplated by many physicists with some dissatisfaction, and with the desire, if possible, to find some explanation or physical means by which so apparently purposeless an end is averted, and of avoiding the necessity for assuming in past time a violation of physical principles at present recognized to exist. The allied notion of an unstable universe whose parts tend to agglomerate together into one mass by successively falling together, would certainly, to

* Communicated by the Author. 
say the least, appear to have something incongruous and unnatural about it, when regarded from a philosophical point of view; and however well grounded this aspect of the case might appear, still, from the vastness of the subject and the limited range of observation, it remains always conceivable that some physical link may have been left out, which affects the tinal conclusion.

As previous attempts to explain the phenomena of nature as the result of the action in the past of existing physical principles have been invariably welcomed, I venture to submit the following conclusion which has presented itself to me. I will commence at once by an illustrative case, noticing the difficulties as they arise. Let us imagine (for mere sake of illustration) a cubical envelope, which permits neither change of volume nor passage of heat, to enclose a space of diameter say $10^{10}$ times the distance between the Sun and Sirius. First, let the matter within this space be at the zero of temperature. Second, let all the matter within our envelope be at such a temperature that it is entirely dissociated into discrete molecules*. Between those two extremes there is room for any number of mean states in which matter might be more or less aggregated or discrete. Might not the universe actually be in one of these intermediate states? $i$. e. consisting of portions of matter in various stages of aggregation, moving among each other according to the principles of the kinetic theory, but not sufficiently rapidly to prevent gravity from producing a certain degree of aggregation. It should be scarcely necessary to observe that we have limited our space merely for the sake of fixing our ideas. All we require is gained if, instead of using the impermeable envelope, we surround our cube with infinite space filled with matter in a similar condition to that which the cube enclosed. There might perhaps be some who wou'd find a difficulty at first sight in conceiving how two masses in translatory motion could collide without gravity making them coalesce and so the degree of aggregation continually becoming greater and greater. But it is to be noted that, if we imagine the masses to collide at such a limiting speed that the velocity with which

* The discrete molecules would of course be in motion, rebounding from each other: in all directions, according to the principles of the kinetic theory of gases, and pervading the cubical unit of volume uniformly. Obviously we must take into account the neutralization of gravity within the cubical unit of volume, by realizing the space outside the cube filled with matter in a similar state to that which the cube encloses. The known tendency of the linetic theory is automatically to produce a similar distribution of matter per unit of rolume. Gravity acting from one unit of volume to another is thus neutralized. 
the fragments or scattered parts rebound or glance off exceeds the greatest velocity of approach that gravity could generate in them when falling together from in indefinite distance, then the degree of aggregation after the collision would be less than it was before. Indeed, fixing the imagination upon a single cubical element of space containing detached solid masses of unatter, and enclosed by a rigid envelope [other similar cubical elements existing outside-and the masses being figured for the instant at rest], it is then quite conceivable that such a velocity might be suddenly given to these detached masses of matter that the heat developed at their mutual collisions is sufficient to resolve the whole into discrete molecules (or a gas) pervading the cubical envelope uniformly, $i$. e. so that gravity is incompetent to produce any degree of aggregation (in the form of clusters or nuclei) at all*. Is not a less velocity than this conceivable which, when communicated to the masses, would still leave them some degree of aggregation, dependent on the mean velocity of translatory motion? which velocity (though constant from one unit of volume to another) would vary greatly from one mass to another in accordance with the principles of the kinetic theory-producing corresponding variations in the degree of aggregation from one mass to another without affecting the mean state (per unit of volume).

The case is comparable on smaller scale to the minute masses (each consisting of a number of molecules aggregated about a common centre) forming the compound molecules of a gas of high complexity. Here it is a known fact that such a velocity of translatory motion (dependent on temperature) might be given to those minute masses (compound molecules) composing the gas as to break them up into discrete molecules. A less velocity than this is conceivable at which a small degree of aggregation is possible, and a still less velocity where a still greater degree of aggregation can ensue. Indeed, if the constituents be numerous so as to admit of a great variety of groupings, a very considerable range in the degree of aggregation is possible by varying the rates of translatory motion (dependent on varying temperatures). It is also a recognized fact here that the degree of aggregation by any given rate of translatory motion (temperature) refers only to the mean state, and not to the state of each of the individual

* It is evident that since we are not limited as to velocity, a certain (adequate) velocity must exist, corresponding to an adequate degree of enercy, that would suffice to produce this result. Of course the cubical envelopes are merely used for facility of illustration, and may be supposed abolished, substituting for them infinite space containing matter in a similar state. 
masses; for frequently one of the minute masses (molecular clusters) may acquire such a velocity in the accidents of collision as to break it up into discrete molecules at an encounter, these discrete molecules grouping together again in another part of the gas, the mean state of aggregation (per unit of volume) remaining unchanged. Of course it is obvious that there are differences of detail in considering the case of the masses of the universe. For example, in a compound gas, where the central agency producing aggregation is " chemical action," the fluctuation in size of a mass (molecular cluster) under the collisions is limited, whereas in the case of the masses of the universe, where the central agency producing aggregation is mainly "gravific action", the fluctuation in size

* The agency producing aggregation may even be found (when recognized) to be of the same kind in all cases " gravific action," "chemical action," \&c.). In former paper's published in the Philosophical Magazine (Sept. and Nov. 1877, and Feb. 1878), I have called attention to the fact that, if the kinetic theory be appplied to the motion of the particles of wther [in addition to that of the stellar masses suggested here], the gravitation of the molecules of gross matter may be accounted fir under the ingenious fundamental idea contained in Le Sage's well-known theory, with the removal of all his postulates; or gravitation may be shown to be the necessary consequence of the mere immersion of the universe in finely subdivided matter moving automatically according to the principles of the kinetic theory. By the application of this theo:y to the stellar masses, molecules of gross matter, and particles of wether, as a vast whole, consisting of matter of different dimensions moving under its own dyuamics, a grand dynamical generalization would evidently seem to suggest itself. It may be noted that, if we reject the now pracically defunct conceptiou of "force" in the sense of an "action at a distance" (without the intervention of matter), no other than a dynamical view of the universe is in principle conceivable.

Another consideration would seem to have an important bearing on this subject. The parts of the molecules of matter are known to possess a considerable capacity for motion. When, for example, the nolecules of a gas are exposed to the pulsatory movement of the waves of heat (from some radiant source), the parts of the molecules are thrown into motion (vibration); and this development of motion in the parts is found to produce an accession of translatory motion in the molecules as wholes. This principle holds independently of scale; and thus it eppears that the development of motion (by any means) in the constituent parts of a mass tends (under certain conditious) to produce translatory motion in the mass as a whole. If the rther, in which gross matter is immersed, be itself in a state of internal motion, this motion must inevitably communicate itself (to a certain extent) to the constituent parts of masses of matter immersed in the æther, and, accordingly, serve as a supplementary means to the development of translatory motion in the masses as wholes.

We may observe that, under the kinetic theory of gravity (based upon Le Sage's fundamental idea), both the range and intensity of gravity have a limit. The range (limited by the length of path of the particles) need not be necessarily much greater than that of which we have proof by otservation, which is but a relatively small range compared with the stellax distances. The intensity of gravity therefore (for this reason) would not 
would not be thus definitely limited. We are, however, dealing here with a fundamental matter of principle, not with subsidiary details*. It appears difficult to see where we are to limit the scale in the application of a principle, or how, if the kinetic theory be applicable to the case of a compound gas consisting of small masses (molecular clusters) of, say, as many as 50 to 60 molecules aggregated about a common centre, it should not apply when the number of molecules aggregated about a centre is increased (so as to form a visible mass). It might be said that the cases are different, inasmuch as the compound molecules of a gas rebound from each other as elastic bodies, whereas in the case of the masses of the universe they would generally be broken up and scattered at the encounters. This objection could only arise, however, from a superficial view of the case. For it is a known fact that the compound molecules of a gas often acquire in the accidents of collisions very great velocities, and they are thus broken up and their parts scattered at their encounters. There is, however, on the whole no work done (or loss of energy) in this breaking-up of the minute masses (compound molecules) of the gas ; for the dissociated molecules unite again in another region of the gas; and so long as the mean state of aggregation (per unit of volume) in the gas remains unchanged, there is on the whole no work done. So in the case of the universe, if the mean state of aggregation remain the same, there would be on the whole no work performed by the occasional breaking-up of matter. But it may be said that at every such collision of two masses of the universe there would be a dissipation of energy in the æther attendant on the heat developed at the encounter, and this energy would be unavoidably lost. But if we regard the matter of the universe as (in the mean) uniformly diffused, as it would necessarily be under the kinetic theory, there would be no such actual loss of energy-merely a radiation backwards and forwards from one region to another. Thus in the smaller scale case of a gas, there is undoubtedly a dissipation of energy in the sother at every encounter of the small-scale masses (molecular clusters) in translatory motion; but this energy is (as is known) not lost, but only radiated to another

go on increasing indefinitely by the imaginary continual piling-up of matter, but would attain a final maximum. We allude to this last point as a possible detail of interest, without thereby implying that it essentially affects the main principle ne have been developing in this paper.

* We merely apply in principle the same general mechanical considerations to molecules aggregated into clusters (lumps) under chemical action, as to molecules aggregated into lumps under gravific action (stellar masses). 
region of the gas, the mean temperature (per unit of volume) of the gas remaining the same. One known consequence of the kinetic theory is that vast fluctuations of temperature may occur in the gas, from one of the portions of matter (consisting of one molecule or several) that moves as a whole in the motion of translation, to another portion, though the whole (reckoned by units of volume) may be in equilibrium of temperature. The same would apply in principle to the case of the universe, consisting of portions of matter in translatory motion among each other, if we do notlimit the scale on which the principles are applicable; or it would follow that there might be vast differences of temperature from one portion of matter (stellar mass) to another, while the whole (reckoned by units of volume) might be in equilibrium of temperature. In order to have an idea, by inspection, of the mean state of temperature of the universe, we should require to sweep over a unit of volume, containing some hundreds of milions of stellar masses (dark and bright), in the same way as we do (on smaller scale) in examining the state of temperature of a gas; any appreciable volume of which contains some hundreds of millions of portions of matter in translatory motion (at different temperatures).

If it were possible to visualize the individual molecules in a compound gas at normal temperature, molecules would be observed in various parts glowing at a white or red heat (and some in a state of dissociation); and if the state of temperature of the whole gas were judged of from these relatively fow luminous molecules, the assumption would be that the whole gas was at a white heat. So in the case of the universe, if the state of temperature of the whole were judged of from the perhaps relatively few luminous masses [which of course can alone be visible to us], an entirely false impression might be conveyed of the real state.

If therefore the above suggestion as to the possible application of an admitted dynamical principle on a large scale should be found valid, it would follow that the universe may already be at uniform temperature, in the sense that the limits within which there is fluctuation of temperature are indefinitely small compared with the collective universe, but that these limits are, relatively to a planetary system, very great, and amply sufficient to allow continual physical change, adapted to the maintenance of life.

So in an analogous way as regards the state of aggregation of the matter of the universe: since, by the application of the kinetic theory, this depends on the temperature, it would follow that the mean state of aggregation of the matter (per unit 
of volume), like the mean temperature, is the same throughout-though indefinite fluctuations of dimensions would occur from one mass to another, in harmony with the fluctuations of velocity.

It would further follow from the principle that molecules of different densities (molecular weights) tend forcibly to become uniformly diffused, that by an adequate past duration of the universe the different kinds of matter must be uniformly diffused (per unit of volume) by the continued interchange of motion, though considerable fluctuations of mixture within ranges less than a unit of volume would be possible in harmony with the kinetic theory.

It may be observed that in principle, in order to account for the continuance of change in the universe, the existence of some process of recurrence is absolutely essential. The cooled down material of extinct suns must in some way be made available for the development of fresh suns or centres of heat. For if this were not the fact, there would be a continual accumulation of the material of extinct or useless suns in the universe, and processes of renewal and maintenance of the activity of the universe would come to a deadlock in the absence of matter to operate upon. It seems inconceivable how this end could be effected under recognized dynamical principles, excepting through an exchange of motion going on among the matter of the universe, involving collisions and an alternating renewal and loss of heat: or this seems on broad principle the only conceivable way in which there should be recurrence, under the condition that the same matter should be used again. There would appear to be a simple grandeur (not out of harmony with the recognized characteristics of nature) in this great result being brought about by the mere movement of the stellar masses according to the kinetic theory. Moreover the kinetic theory has been mathematically proved (when a large number of masses are concerned) to produce a system of order and symmetry (or mean similarity of the conditions in all parts of the system) which is rigidly and automatically maintained by a process of self-correction under dynumical principles-a self-acting adjustment of the motions continually taking place, whereby a system of harmony and order is maintained everywhere, a perfect state of mobile equilibrium existing in all parts.

'Those who are inclined to view the physical causation of the past in the light of the physical causation of the present, or who look upon the principle of the conservation of onergy as a truth as nocessary in the past as in the present (or who are disposed to regard physical truths as independent of time), 
are bound to believe that some process of recurrence must exist, whereby useful change and activity are continned in the universe, and the purposeless end of a changeless chaos prevented-and that we should seek for the explanation of this, not so much with the view to prove the fact thereby, but rather as a satisfaction or confirmation of a fact we already had logical grounds for believing to exist.

That recurring changes exist in the universe seems to have been the conviction of Sir W. Grove. He remarks relative to this subject ("Corr. of Physical Forces,' page 67):-"Enlarged observation may prove that phenomena seeming to tend in one direction will turn out to be recurrent, though never absolutely identical in their recurrence; that there is throughout the universe gradual change, but no finality; .... that no star or planet could at any time be said to be created or destroyed, or to be in a state of absolute stability, but that some may be increasing, others dwindling away, and so throughout the universe, in the past as in the future."

Humboldt also says, as regards this point (Preface to 'Cosmos"):- "I would therefore venture to hope that an attempt to delineate nature in all its vivid animation and exalted grandeur, and to trace the stable amid the vacillating ever-recurring alternation of physical metanorphoses, will not be wholly disregarded at a future age."

It has been pointed out by Dr. James Croll, in a paper published in the Quarterly Journal of Science for July 1877, that Helmholtz's gravitation theory of the origin of the sun's heat is not alone sufficient to account for a past duration of the sun's heat in harmony with geological evidence as to tho age of the earth. It is of course evident that all the geological history of the earth must be comprised within the limit of the age of the sun. In this paper elaborate geological evidence is given tending to prove conclusively that as a limit, the age of the earth must be very much (at least three times) greater than the time the store of heat could have existed in the sun, if the origin of this heat were solely gravitation. For it is a known fact (based upon direct experimental evidence of the loss of solar heat by radiation) that the store of heat, if it had resulted from gravitation alone, would only have sufficed for about 20,000,000 years: Dr. Croll remarks regarding this point (page 317):-- " We have not sufficient data to determine how many years have elapsed since life began on the globe, for we do not know the total amount of rock removed by denudation; but we have data perfectly sufficient to show that it began far more than twice 20 million years ago. ..... Now in proving that tho antiquity of our habitable globe must 
be far greater than 20 or 30 million years, we prove that there must have been some other source in addition to gravity from which the sun derived his store of energy." The collision of matter in translatory motion is then suggested as the only conceivable other source of the store of heat-energy in the sun-this suggestion having already been made by the same author in a previous paper published in the Philosophical Magazine for May 1868, where it is remarked (p. 373):" The Dynamical Theory of Heat affords an easy explanation of at least how such an amount of energy may have been communicated. Two bodies, each one half the mass of the sun, moving directly towards each other with a velocity of 476 miles per second, would by their concussion generate in a single moment 50,000,000 years' heat" [i.e. an amount of heat which would cover the present rate of the sun's radiation for a period of $50,000,000$ years].

It would seem to be scarcely realized what a field for rapidity of motion combined with all the stability or permanence of apparent rest the universe presents. It is a mere question of scale for bodies to possess any velocities (no consequence how great), and yet not alter their relative positions appreciably in a given epoch of time. Thus a stellar mass, for example, that moved transversely to the observer a distance equal to its own diameter [say a million miles, which is roughly the sun's diameter] in a second, would appear to the eye to be at rest; for the disk of the stellar mass has no apparent diameter even in the best telescope, and therefore the distance moved in a second would be invisible. Yet the velocity of the stellar mass in that case would be about five times that of light*. Dr. Croll has pointed out (Phil. Mag. July 1878) that

* Since light requires a quarter of an hour to traverse the diameter of the earth's orbit, and since that diameter (182,000,000 miles) would be a point when viewed at the distance of the nearest star, it follows that, if the nearest star were moving transversely to the observer with the velocity of light, the star might be watched for a quarter of an hour without appearing to deviate from its position. The actual angular distance traversed by the star, after the motion equal to that of light had been going on for a quarter of an hour, would be the thickness of a human hair held at 25 feet from the eye [this being the known representation of an angle of 2 seconds, which is that subtended by the earth's orbit at the distance of the nearest star]. This star might move with the velocity of light for $7 \frac{1}{2}$ hours without traversing a greater angular distance than the thickness of a hair held 10 inches from the eye.

The tendency of modern science is unquestionably to look to a dynamical interpretation of phenomena in place of the old vaguely conceived statical ideas. The old tendency bas been rather in the direction of ignoring the motions of the stellar masses, and of banishing from the conceptions (in the attempt to arrive at some notion of stability in the universe) all idea of a direct interference or mutual action of the moving parts of the 
a star 1000 times more remote than $\alpha$ Centauri, though moving transversely to the observer at the rate of 100 miles per second, would take upwards of 30 years to change its position as much as $1^{\prime \prime}$-in fact, that we should have to watch the st..r for a generation or two before we could be certain whether it was changing its position or not. It is evident that in regard to the motions of the stellar masses, some attempt mut be made to realize their dimensions in order to have a just appreciation of the case. A motion that might seem harmonious and even slow in the case of a great ship (for examp'e) would be utterly discordant and unfitting in the case of a child's toy boat. A stellar mass that moved through a fractional thousandth part of its own diameter in a second might be regarded as having a majestically slow motion; and yet if its diameter were a million miles [which may possibly be an average value], it would have traversed a thousand miles in that brief interval of time. These considerations may serve to show that if we apply the kinetic theory to the motions of the stellar masses, how completely different the case is from an ordinary gas as regards sequence of changes. The molecules of a gas, so far from merely traversing a space comparable to their own diameters in a second, are known as an actual fact to traverse a space equal to countless millions of times their own mean distance in a second [making roughly about ten thousand million collisions a second]; and yet, in spite of this rapid sequence of changes, the molecules, as regards absolute velocity, are almost at rest compared with the stellar masses. The scale is so incomparably different in the two cases*. Nevertheless, from the fact that the energy (heat) developed at the collisions depends on the absolute velocity, and of course not on the relative change of position of the masses, it becomes thereby possible by a sufficient scale to have an absolute velocity of any value however high, adequate to an enormous development of heat and explosive rebound $\dagger$ at the encounters, combined with so small a relative motion (or relative change of position) that the masses appear

universe upon each other. We, on the other hand, are led to take the diametrically opposite view, and to look to the dynamical acticn of the moving parts of the universe upon each other as the sole means of ensuring stability.

* While in the case of a gas the scale is too small for us to overlook directly the changes taking place in a unit of volume, in the case of the universe the scale is too big.

† The rebound would be far more than "explosive" in the ordinary sense of that term, since the expansive action of the heat developed by collisions even at moderate planetary velocities (calculably) far outrivals both in energy and suddenness the case of explosives. Indeed, if the colliding masses were formed of gunpowder, its ignition at the collision would add but little to the explosive energy of the recoil. 


\section{On the Continuance of Recurring Changes in the Universe.}

to the eye to be at rest. If it takes so long for the stellar masses to change their mean distances, what must it be in regard to the time taken to traverse their mean length of path (previous to the encounters); and thus in each individual case an almost limitless epoch of time is rendered possible for the conditions of life, while in the collective universe that stability and permanence are ensured which can alone rest upon recurring change.

In regard to a possible objection that, as far as observation has gone, the proper motion of the stars has not been found to exceed 30 to 50 miles per second, it may be replied that those stars whose proper motions it has been possible to estimate roughly, constitute an insignificant and almost vanishing minority compared with the rest of the visible stars, which are known to be situated at immeasurable distances. It has been pointed out by Dr. Croll that it would be scarcely reasonable to expect luminous stars to possess a high proper motion, since precisely they would have lost a greater part of their proper motion in the collisions which developed the heat which rendered them luminous, the proper motion having been lost by conversion into heat. Mr. Johnstone Stoney, in a paper published in the Proceedings of the Royal Society for 1868-69, has also dealt with the eventuality of collisions among the stars in a state of proper motion, and remarks (page 53):- "If what I here venture as a surmise with respect to the proximate cause of stellar heat and the origin of double stars is what really took place, we must conclude the sky to be peopled with countless hosts of dark bodies, so numerous that those which have met with such collisions as to render them now visibly incandescent must be in comparison few indeed" *

The occasional appearance or blazing forth of " new stars," so notorious in astronomy, as if due to some sudden convulsion, would be in harmony with the view of collisions. That the stellar masses are in translatory motion, moving among each other in various directions and (in general) at such distances apart that gravity [if it exists at all at such distances] is incompetent to prevent the paths from being (sensibly) straight lines, is a well-established fact of observation. Tho application of the principles of the kinetic theory to the case would, therefore, seem to suggest itself rather in the light of a natural de-

* The fact of the present writer having arrived at the general conclusions enunciated in this article, before he had seen the papers of Dr. Oroll and Mr. Johnstone Stoney, served rather to increase his confidence in the view he had adopted, and which he now ventures to suggest to the readers of the Philosophical Magnzine. 
duction than as a mere speculation. And even independently of all other considerations, it would seem more reasonable to look to a dynamical interpretation of the motions of the stellar masses, than to regard them as drifting indiscriminately among each other with the absence of all recognized purpose.

London, June 1870.

XX. On the Theory of Faults in Cables. By Oliver Heaviside.

[Concluded from p. 74.]

16. THERE is no difficulty in finding formula from the preceding results which will correspond to any particular example considered. Such formulæ, however, have, save to the mathematically curious, little value or interest unless they are interpreted numerically. Even then the labour involved is, save in special cases, ont of proportion to the derived benefit. I shall confine myself to the simple cases of direct working without condensers, and with condensers, with a single fault in the centre of the line.

Suppose the signalling is made by means of a battery at $\mathrm{P}$ and a receiving instrument at $Q$, both of negligible resistance, and to earth direct. Then

$$
0=m_{1}=m_{2}=n_{1}=n_{2} \text {. }
$$

Let there be a single fault of resistance $\approx k l$ at the centre of the line. Then

$$
\begin{aligned}
\tan b & =0, \\
\sin a+\frac{1}{z a} \sin ^{2} \frac{a}{2} & =0,
\end{aligned}
$$

by (10) and (11). The latter splits up into

$$
\sin \frac{a}{2}=0 \text { and } \tan \frac{a}{2}=-2 z a . \quad \text {. . . }
$$

Therefore, when $i$ is even, $a_{i}=i \pi$; and when $i$ is odd, $a_{i}$ lies between $i \pi$ and $(i+1) \pi$. The denominator of $(19)$ is

$$
\begin{aligned}
\phi(a, b) & =\int_{0}^{\frac{l}{2}} \sin ^{2} \frac{a x}{l} d x+\int_{\frac{l}{2}}^{l}\left\{\sin \frac{a x}{l}+\frac{\sin \frac{a}{2}}{z a} \sin a\left(\frac{x}{l}-\frac{1}{2}\right)\right\}^{2} d x \\
& =\frac{l}{2}\left(1-\frac{\sin a}{a}\right) .
\end{aligned}
$$

Therefore, by (20),

$$
A_{i}=\frac{2 \mathrm{~F}_{i}}{a^{i}-\sin \alpha_{i}} .
$$

